Pacific Journal of Mathematics

DECOMPOSING MODULES INTO PROJECTIVES AND 


\title{
DECOMPOSING MODULES INTO PROJECTIVES AND INJECTIVES
}

\author{
P. F. SMITH
}

A ring $R$ is called a right PCI-ring if and only if for any cyclic right $R$-module $C$ either $C \cong R$ or $C$ is injective. Faith has shown that right PCI-rings are either semiprime Artinian or simple right semihereditary right Ore domains. Thus if $R_{1}$ and $R_{2}$ are right PCI-rings then $R=R_{1} \oplus R_{2}$ is not a right PCI-ring unless $R_{1}$ and $R_{2}$ are both semiprime Artinian but $R$ has the property that every cyclic right $R$ module is the direct sum of a projective right $R$-module and an injective right $R$-module, and rings with this property on cyclic right $R$-modules will be called right CDPI-rings. On the other hand, if $S$ is a semiprime Artinian ring then the ring of $2 \times 2$ upper triangular matrices with entries in $S$ is also a right CDPI-ring. The structure of right Noetherian right CDPI-rings is discussed. These rings are finite direct sums of right Artinian rings and simple rings. A classification of right Artinian right CDPI-rings is given. However the structure of simple right Noetherian right CDPI-rings is more difficult to determine precisely and the problem of finding it reduces to a conjecture of Faith.

1. Introduction. Recall that if $X$ is a nonempty subset of a ring $R$ (and by a ring we shall always mean a ring with identity element) then the left annihilator of $X$ is the set of all elements $r$ of $R$ such that $r x=0$ for every element $x$ of $X$, and is denoted by $l(X)$. Similarly the right annihilator of $X$ is $r(X)=\{r \in R: x r=0$ for all $x$ in $X$ \}. A subset $A$ of $R$ is called a left (respectively right) annihilator in case $A=l(X)(A=r(X))$ for some nonempty subset $X$ of $R$. A ring $R$ is a Baer ring if and only if for every right annihilator $A$ in $R$ there exists an idempotent element $e$ such that $A=e R$, equivalently for every left annihilator $B$ in $R$ there exists an idempotent element $f$ such that $B=R f$. Examples of Baer rings can be found in [6]. Baer rings are examples of right PP-rings, that is rings such that every principal right ideal is projective. On the other hand, Small [9], Theorem 1, showed that if $R$ is a right PP-ring and $R$ does not contain an infinite collection of orthogonal idempotents then $R$ is a Baer ring.

A right CDPI-ring $R$ is a right PP-ring (in fact it is right semihereditary, see [10], Lemma 2.4) and has the property that $R / E$ is an injective right $R$-module for every essential right ideal $E$ of $R$ (see Corollary 2.2). Rings with this latter property we shall call right RIC-rings ("RIC" for restricted injective condition). If a ring 
$R$ is a Baer ring, then $R$ is a right CDPI-ring if and only if $R / E$ is an injective right $R$-module for every right ideal $E$ of $R$ with zero left annihilator (Theorem 2.4). Recall that Osofsky [8] proved that a ring $R$ is semiprime Artinian if and only if every cyclic right $R$ module is injective.

A ring $R$ is a right CEPI-ring provided every cyclic right $R$ module is the extension of a projective right $R$-module by an injective right $R$-module. The class of right CEPI-rings coincides with the class of right PP - right RIC-rings (Theorem 2.9) but strictly contains the class of right CDPI-rings since there is an example in [10] of a right and left Artinian right and left CEPI-ring which is not a right CDPI-ring.

Let us call a ring $R$ a right $P C I$-domain provided $R$ is a right PCI-ring and a domain. Goodearl [5] called a ring $R$ a right SI-ring in case every singular right $R$-module is injective. By [10], Corollary 4.8, if $R$ is a right Noetherian right CDPI-ring then $R$ is a right SI-ring and hence by [5], Theorem 3.11, and [3], Theorems 14 and 17, $R$ is a finite direct sum $A \oplus B_{1} \oplus B_{2} \oplus \cdots \oplus B_{n}$ where $A$ is a right Artinian right CDPI-ring and for each integer $1 \leqq i \leqq n$, the ring $B_{i}$ is a right CDPI-ring Morita equivalent to a right Noetherian simple right PCI-domain, and conversely. The ring $A$ can be characterized as a certain ring $(S, M, 0, T)$ of $2 \times 2$ "matrices"

$$
\left[\begin{array}{rr}
s & m \\
0 & t
\end{array}\right]
$$

with $s$ in a semiprime Artinian ring $S, t$ in a semiprime Artinian ring $T$ and $m$ in a certain left $S$-, right $T$-bimodule $M$, under the usual matrix addition and multiplication (Corollary 3.8).

When it comes to the rings $B_{i}(1 \leqq i \leqq n)$ the natural question which arises is the following one.

Question 1.1. Given a right Noetherian simple right PCI-domain $D$, is any ring $S$ Morita equivalent to $D$ a right CDPI-ring?

This question is related to a conjecture of Faith [3], p. 111, and to show the connection between them we make the following definitions. Let $m$ be a positive integer. A ring $R$ is a right FGDPI-ring (right $F G D P I_{m}-$ ring) if and only if every finitely generated ( $m$-generator) right $R$-module is the direct sum of a projective right $R$-module and an injective right $R$-module. Right Noetherian semiprime right FGDPI ${ }_{2}$-rings are right FGDPI-rings and are left Goldie (Theorem 5.7). It follows that (see Corollary 4.12) the answer to 1.1 is "yes" if and only if $D$ is a left Ore domain and this is precisely Faith's conjecture, and in this case the rings $B_{i}(1 \leqq i \leqq n)$ are just the rings Morita 
equivalent to right Noetherian simple right PCI-domains. Recall that if the ring $D$ is a left Ore domain then Faith [3], Theorem 22 and subsequent remarks, proved that $D$ is a left Noetherian left PCI-domain and we call such rings Noetherian simple PCI-domains. Examples of these rings can be found in [2]. Faith's conjecture can be expressed in yet another way (see Theorems 4.11 and 5.7):

Conjecture 1.2. If $D$ is a right Noetherian simple right PCIdomain then the ring $D_{2}$ is a right CDPI-ring where $D_{2}$ is the complete ring of $2 \times 2$ matrices with entries in $D$.

We shall call a ring $R$ a Noetherian simple PCI-domain if and only if $R$ is a right and left Noetherian simple right and left PCIdomain. Examples of Noetherian simple PCI-domains have been produced by Cozzens [2]. For any positive integer $m$ a ring $R$ is a right Noetherian right FGDPI $_{m}$-ring if and only if $R$ is a finite direct sum $A \oplus B_{1} \oplus B_{2} \oplus \cdots \oplus B_{n}$ where $A$ is a right Artinian right FGDPI $_{m}$-ring and for each integer $1 \leqq i \leqq n$ the ring $B_{i}$ is a simple right and left Noetherian ring Morita equivalent to a Noetherian simple PCI-domain (see Corollary 5.8). There is a corresponding structure theorem for right Noetherian right FGDPIrings. We have not been able to find explicitly the structure of right Artinian right FGDPI $_{m}$-rings ( $m$ an integer greater than 1) or right Artinian right FGDPI-rings.

We mention one further interesting fact about semiprime rings. If $R$ is a semiprime ring then the following statements are equivalent:

(i) $R$ is a right Noetherian right FGDPI $_{2}$-ring,

(ii) $R$ is a left Noetherian left FGDPI ${ }_{2}$-ring,

(iii) $R$ is a right Noetherian right FGDPI-ring, and

(iv) $R$ is a left Noetherian left FGDPI-ring (see Corollary 5.9). Note also that if $R$ is a right Noetherian right FGDPI $_{2}$-ring then $R$ is a left SI-ring and in particular $R$ is left hereditary (see Corollary 5.10).

2. Right CDPI-rings. In this section we first look at characterizations of right CDPI-rings, we then examine the relationship between right CEPI-rings and right RIC-rings and finally we generalize the theorem of Osofsky mentioned in the Introduction.

Lemma 2.1 (See [10], Lemma 5.1). A ring $R$ is a right CDPIring if and only if for every right ideal $E$ of $R$ there exists an idempotent element $e$ such that $E$ is contained in the right ideal $e R$ and the right $R$-module $e R / E$ is injective. 
ideal of $R$ with zero left annihilator. Then the right $R$-module $R / E$ is injective.

If $X$ is a nonempty subset of a ring $R$ then by $r l(X)$ we shall mean $r(l(X))$, the right annihilator of the left annihilator of $X$. The proof of the next result is an easy adaptation of the proof of [10], Lemma 5.7 .

LEMMA 2.3. Let $R$ be a Baer ring. Then $R$ is a right CDPIring if and only if $r l(E) / E$ is an injective right $R$-module for each right ideal $E$ of $R$.

THEOREM 2.4. Let $R$ be a Baer ring. Then $R$ is a right CDPIring if and only if $R / E$ is an injective right $R$-module for each right ideal $E$ of $R$ with zero left annihilator.

Proof. In view of Corollary 2.2 we need prove only the sufficiency. Suppose that $R$ is a ring such that $R / E$ is injective for every right ideal $E$ with $l(E)=0$. Let $A$ be a right ideal of $R$. Since $R$ is a Baer ring there exists an idempotent element $a$ of $R$ such that $r l(A)=a R$. Let $B=\{r \in R: a r \in A\}$. Since $a$ is idempotent it follows that $A=a A$ and hence $A \subseteq B$. Then $a R=r l(A) \subseteq r l(B)$. But $(1-a) R \subseteq B \subseteq r l(B)$ and hence $\operatorname{Rrl}(B)$. Thus $l(B)=0$ and by hypothesis $R / B$ is injective. Since the mapping $\varphi: R / B \rightarrow \alpha R / A$ defined by $\varphi(r+B)=a r+A(r \in R)$ is an $R$-isomorphism it follows that $a R / A$ is injective. By Lemma $2.1 R$ is a right CDPI-ring.

COROLLARY 2.5. Let $R$ be a ring which does not contain an infinite collection of orthogonal idempotent elements. Then $R$ is a right CDPI-ring if and only if $R$ is a right $P P$-ring and $R / E$ is an injective right $R$-module for every right ideal $E$ of $R$ with zero left annihilator.

Proof. The necessity is a consequence of Corollary 2.2 and [10], Lemma 2.4. The sufficiency follows by the theorem and [9], Theorem 1.

An immediate consequence of Corollary 2.5 is the next result.

COROLLARY 2.6. Let $R$ be a semiprimary ring. Then $R$ is a right CDPI-ring if and only if $R$ is a right PP-ring such that $R / E$ is an injective right $R$-module for every right ideal $E$ of $R$ with zero left annihilator.

CoROLlaRy 2.7. Let $R$ be a ring which does not contain an 
infinite direct sum of nonzero right ideals. Then $R$ is a right CDPI-ring if and only if $R$ is a right nonsingular ring such that $R / E$ is an injective right $R$-module for every right ideal $E$ of $R$ with zero left annihilator.

Proof. The necessity follows by Corollary 2.2 and [10], Lemma 2.4. Conversely, suppose that $R$ is a right nonsingular ring such that $R / E$ is an injective right $R$-module for each right ideal $E$ with $l(E)=0$. Since $R$ is right nonsingular it follows that $R$ is a right RIC-ring. Also by [4], Lemma 1.4 and Theorem 2.3 (iii), $R$ is a right Goldie ring. By [10], Corollary 4.3 and Lemma $4.4, R$ is a right PP-ring. Finally by Corollary $2.5 R$ is a right CDPI-ring.

Next we consider briefly right CEPI-rings. Let $E$ be a right ideal of a right CEPI-ring $R$. There exists a right ideal $F$ of $R$ containing $E$ such that $F / E$ is projective and $R / F$ is injective. Since $F / E$ is projective there exists a right ideal $G$ of $R$ such that $E \cap$ $G=0$ and $F=E \oplus G$. Moreover, $G \cong F / E$ is projective. We have proved:

LEMMA 2.8. A ring $R$ is a right CEPI-ring if and only if for every right ideal $E$ of $R$ there exists a projective right ideal $G$ of $R$ such that $E \cap G=0$ and $R /(E \oplus G)$ is an injective right $R$ module.

In [10], Lemma 2.4, we proved that a right CEPI-ring is a right semihereditary right RIC-ring. Now we have the following result.

TheOREM 2.9. A ring $R$ is a right CEPI-ring if and only if $R$ is a right $P P$ - right $R I C$-ring.

Proof. As we have just remarked the necessity is proved in [10], Lemma 2.4. Conversely, suppose that $R$ is a right PP-right RIC-ring. Let $E$ be a right ideal of $R$. By Zorn's lemma there exists a maximal collection $S$ of nonzero elements $x_{\lambda}(\lambda \in \Lambda)$ of $R$ such that if $H=\sum x_{\lambda} R$ then $H=\bigoplus_{A} x_{\lambda} R$ and $E \cap H=0$. Since $R$ is a right PP-ring, $H$ is projective. Let $a$ be a nonzero element of $R$. If $a \notin S$ then either $a R \cap H \neq 0$ or $E \cap(a R \oplus H) \neq 0$. It follows that $E \oplus H$ is an essential right ideal of $R$. Since $R$ is a right RIC-ring, the right $R$-module $R /(E \oplus H)$ is injective. By Lemma 2.8 $R$ is a right CEPI-ring.

Finally in this section we give the following generalization of Osofsky's theorem [8]. 
THEOREM 2.10. A ring $R$ is semiprime Artinian if and only if $R$ is a right self-injective right $R I C$-ring.

Proof. The necessity is a consequence of Osofsky's theorem. Conversely, let $R$ be a right self-injective right RIC-ring. Since $R$ is right self-injective, given any right ideal $A$ of $R$ there exists an idempotent element $e$ of $R$ such that $A$ is an essential submodule of the right ideal $e R$. Since $R$ is a right RIC-ring it follows that $e R / A$ is injective. By Lemma $2.1 R$ is a right CDPI-ring. Let $C$ be a cyclic right $R$-module. There exists a projective module $P$ and an injective module $Q$ such that $C=P \oplus Q$. Since $P$ is therefore cyclic it follows that $P$ is isomorphic to a direct summand of $R$ and hence $P$ is injective. Thus $C$ is injective. Thus every cyclic right $R$-module is injective and $R$ is semiprime Artinian by Osofsky's theorem [8].

3. Semiprimary right CDPI-rings, Right CDPI-rings are right RIC-rings (see [10], Lemma 2.4). In addition, by [10], Lemma 2.5 and Theorem 4.1, semiprimary right RIC-rings are right SI-rings. Also, by [5], Proposition 3.5, semiprimary right SI-rings are left SI-rings. Thus we have the following result.

LEMMA 3.1. Semiprimary right CDPI-rings are right and left SI-rings.

Let $R$ be a right SI-ring. By [5], Proposition 3.3, $R$ is right hereditary. If in addition $R$ is semiprimary then $R$ is a Baer ring by [9], Theorem 1. Noting this fact, the next result of this section is proved by adapting the proof of [10], Theorem 5.13.

LEMMA 3.2. A ring $R$ is a semiprimary (right) SI-ring if and only if $R$ is semiprime Artinian or there exist semiprime Artinian rings $S$ and $T$ and a left $S$-, right $T$-bimodule $M$ such that $M$ is a faithful left $S$-module and $R$ is isomorphic to the ring $(S, M, 0, T)$.

For the remainder of this section we shall fix the following notation: $S$ and $T$ are semiprime Artinian rings, $M$ is a left $S$-, right $T$-bimodule (not necessarily faithful as a left $S$-module) and $R$ is the ring $(S, M, 0, T)$. That is, $R$ consists of all "matrices"

$$
(s, m, 0, t)=\left[\begin{array}{rr}
s & m \\
0 & t
\end{array}\right]
$$

with $s$ in $S, m$ in $M$ and $t$ in $T$, addition and multiplication in $R$ being the usual matrix addition and multiplication. For each nonempty subset $X$ of $M$ let $\operatorname{Ann}_{S}(X)$ denote the annihilator of $X$ in 
$S$; that is, $\operatorname{Ann}_{S}(X)=\{s \in S: s X=0\}$. Let $I=\operatorname{Ann}_{S}(M)$ and let $q$ be the central idempotent element of $S$ such that $I=S q$. The right socle of $R$ will be denoted by $A$. It can easily be checked that $A=(I, M, 0, T)$ and $A$ is an essential right ideal of $R$. By [5], Proposition 3.1, $R$ is a right SI-ring and in view of Lemma 3.2 we can take $R$ as a typical semiprimary right SI-ring. The Jacobson radical of $R$ will be denoted by $J$. Clearly $J=(0, M, 0,0)$. Moreover $A=J \oplus e R$ where $e$ is the idempotent $(q, 0,0,1)$ of $R$ (here 1 is the identity element of the ring $T$ ). Note that $e J=0$ and recall that $A=\cap\{E: E$ is an essential right ideal of $R\}$.

LEMMA 3.3. Let $R$ be a semiprimary right SI-ring with Jacobson radical $J$ and let $X$ be a right $R$-module. Then $X$ is injective if and only if given any homomorphism $\varphi: J \rightarrow X$ there exists an element $x$ of $X$ such that $\varphi(j)=x j$ for every element $j$ of $J$.

Proof. The necessity is an immediate consequence of Baer's criterion for injectivity (see for example [1], Lemma 18.3). Conversely, suppose that $X$ has the stated property. By Lemma 3.2 we can suppose without loss of generality that in the above notation $R=$ $(S, M, 0, T)$. Let $Z=Z(X)$ be the singular submodule of $X$. Since $R$ is a right SI-ring it follows that $Z$ is injective and hence there exists a submodule $Y$ of $X$ such that $X=Z \oplus Y$. Note that $Y$ is nonsingular. Let $E$ be an essential right ideal of $R$ and $\varphi: E \rightarrow Y$ be an $R$-homomorphism. Let $\alpha$ be the restriction of $\varphi$ to $J$. By hypothesis there exists an element $x$ of $X$ such that $\alpha(j)=x j(j \in J)$. If $x=z+y_{1}$ where $z \in Z, y_{1} \in Y$, then clearly $\alpha(j)=y_{1} j(j \in J)$. Let $y_{2}$ be the element $\varphi(e)$ of $Y$, where $e=(q, 0,0,1)$ as above. Let $y$ be the element $y_{1}(1-e)+y_{2} e$ of $Y$. If $a \in A$ then $a=j+e r$ for some elements $j$ of $J$ and $r$ of $R$ and

$$
\varphi(a)=\varphi(j)+\varphi(e) e r=y_{1} j+y_{2} e r=y a .
$$

Thus $\varphi(a)=y a(a \in A)$. Now let $b \in E$. Since $A$ is an essential submodule of $E$ there exists an essential right ideal $K$ of $R$ such that $b K \subseteq A$. For any element $k$ of $K, \varphi(b) k=\varphi(b k)=y b k$ and hence $(\varphi(b)-y b) k=0$. It follows that $(\varphi(b)-y b) K=0$. Since $Y$ is nonsingular it follows that $\varphi(b)=y b$. Hence $\varphi(b)=y b(b \in E)$, and by Baer's criterion $Y$, and hence $X$, is injective.

It is clear from the proof of Lemma 3.3 that in Lemma 3.3 we can replace $J$ be the right socle $A$.

In view of Corollary 2.6 interest centres on right ideals of $R$ with zero right annihilator. Let $E$ be a right ideal of $R$. Let $F=$ $\{a \in S:(a, 0,0,0) \in E\}$. Then $F$ is a right ideal of $S$ and there exists 
an idempotent element $f$ of $S$ such that $F=f S$. If $\bar{f}$ is the element $(f, 0,0,0)$ of $R$ then $\bar{f} R=(f S, f M, 0,0)$. If $N$ is the $T$-submodule $(1-f) M$ then $M=f M \oplus N$ and $E=\bar{f} R \oplus E_{1}$ where $E_{1}$ is the right ideal $E \cap(0, N, 0, T)$. For, if $r=(a, b, 0, c) \in E$ with $a$ in $S, b$ in $M$ and $c$ in $T$ then $(a, 0,0,0)=(a, b, 0, c)(1,0,0,0) \in E$ and hence $a=f a$ and $r-\bar{f} r \in E_{1}$. Now $E_{1}=\left(E_{1} \cap J\right) \oplus C$ for some right ideal $C$ contained in $E_{1}$. Let $D=\{t \in T:(0, y, 0, t) \in C$ for some element $y$ of $M$ \}. Then $D$ is a right ideal of $T$ and there exists an idempotent element $g$ of $T$ such that $D=g T$. Let $m$ be an element of $M$ such that $c=(0, m, 0, g) \in C$. For any element $c_{1}$ of $C$ it can easily be checked that $c_{1}-c c_{1} \in C \cap J=0$. It follows that $c$ is an idempotent element of $R$ and $C=c R$. In particular $c$ idempotent implies that $m=m g$. Thus there exists a $T$-submodule $X$ of $N$ such that $E$ consists of all "matrices" $(f a, f b+x+m t, 0, g t)$ with $a$ in $S, b$ in $M, x$ in $X$ and $t$ in $T$. Now suppose that $l(E)=0$. It can easily be checked that if $e$ is an idempotent element of $S$ such that $\operatorname{Ann}_{S}(x)=S e$ then $X=(1-f) X$ implies that $e(1-f) \in S e$ and

$$
(e(1-f),-e(1-f) m, 0,1-g)
$$

belongs to $l(E)$. Thus $e(1-f)=0$ and $g=1$. But $e(1-f)=0$ implies that $e=e f$ and $S e \subseteq S f$. This gives the following result after a little checking.

LEMMA 3.4. A right ideal $E$ of the above ring $R$ has zero left annihilator if and only if there exists a T-submodule $X$ of $M$, an idempotent element $e$ of $S$ such that $S e=\operatorname{Ann}_{S}(X)$, an idempotent element $f$ of $S$ such that $S e \subseteq S f$, and an element $m$ of $M$ such that $E$ consists of all "matrices" ( $f a, f b+x+m t, 0, t)$ with $a$ in $S, b$ in $M, x$ in $X$ and $t$ in $T$.

LEMMA 3.5. If $X=\operatorname{Ann}_{M}\left(\operatorname{Ann}_{S}(X)\right)$ for every T-submodule $X$ of $M$ then $R$ is a right CDPI-ring.

Proof. By $\mathrm{Ann}_{M}\left(\mathrm{Ann}_{S}(X)\right)$ we mean the set of elements $m$ of $M$ such that $\operatorname{Ann}_{S}(X) m=0$. In the notation of the previous lemma let $E$ be the right ideal of all "matrices" $(f a, f b+x+m t, 0, t)$ with $a$ in $S, b$ in $M, x$ in $X$ and $t$ in $T$. Let $s \in A_{S n}(f M+X)$; then $s f M=s X=0$. But $s X=0$ implies that $s=s e$ and hence $s f=s e f=$ $s e=s$. It follows that $s M=0$ and hence $A_{n} n_{S}(f M+X)=\operatorname{Ann}_{S}(M)$. By hypothesis $f M+X=\operatorname{Ann}_{M}\left(\operatorname{Ann}_{S}(f M+X)\right)=M$. It follows that the ideal $(0, M, 0, T)$ is contained in $E$. Let $\varphi: J \rightarrow R / E$ be an $R$ homomorphism. If $b=(0,0,0,1)$ then $j=j b$ for every element $j$ of $J$ and it follows that $\varphi=0$. By Corollary 2.6 and Lemmas 3.2-3.4 $R$ is a right CDPI-ring. 
In particular if $S=M=T$ then $R$ is a right CDPI-ring. This special case was proved in [10], Theorem 5.15. Another special case is when $M$ is a simple right $T$-module and again $R$ is a right CDPIring. This corresponds to the Jacobson radical $J$ of $R$ being a minimal right ideal (see [10], Theorem 5.9). We can express Lemma 3.5 in terms of $J$ as follows.

COROLLARY 3.6. Let $R$ be a semiprimary right SI-ring such that $F=J \cap r l(F)$ for every right ideal $F$ contained in the Jacobson radical $J$ of $R$. Then $R$ is a right CDPI-ring.

THEOREM 3.7. In the above notation let $R$ be the semiprimary right SI-ring $(S, M, 0, T)$. Then $R$ is a right $C D P I$-ring if and only if for every $T$-submodule $X$ of $M$ such that $\operatorname{Ann}_{S}(X)=\operatorname{Ann}_{S}(M)$ and every T-homomorphism $\varnothing: M \rightarrow M / X$ there exists an element $a$ of $S$ such that $\varphi(m)=a m+X$ for all $m$ in $M$.

Proof. Suppose first that $R$ is a right CDPI-ring. Let $X$ be a $T$-submodule of $M$ such that $\operatorname{Ann}_{S}(X)=\operatorname{Ann}_{S}(M)=S q$ and $\varphi: M \rightarrow$ $M / X$ a $T$-homomorphism. Let $V$ be a set of coset representatives of $X$ in $M$ and define a mapping $\tau: M \rightarrow V$ by $\varphi(m)=\tau(m)+X(m \in M)$. Let $E$ be the right ideal $(S q, X, 0, T)$. It can easily be checked that $l(E)=0$ and thus, by Corollary $2.2, R / E$ is an injective right $R$ module. Define $\bar{\varphi}: J \rightarrow R / E$ by $\bar{\varphi}(0, m, 0,0)=(0, \tau(m), 0,0)+E(m \in M)$. Since $\bar{\varphi}$ is an $R$-homomorphism there exists an element $r=(a, b, 0, c)$ of $R$ such that $\bar{\phi}(j)=r j+E(j \in J)$. It can easily be checked that this gives $\varphi(m)=a m+X(m \in M)$.

Conversely, in the notation of Lemma 3.4 let $E$ be the right ideal of $R$ consisting of all "matrices" $(f a, f b+x+m t, 0, t)$ with $a$ in $S, b$ in $M, x$ in $X$ and $t$ in $T$. Let $Y$ be the $T$-submodule $f M+X$ of $M$ and let $H$ be the right ideal consisting of all "matrices" $(0, y+m t, 0, t)$ with $y$ in $Y$ and $t$ in $T$. By [5], Proposition 3.3, $R$ is right hereditary. Thus to prove that $R / E$ is an injective right $R$-module it is sufficient to prove that $R / H$ is an injective right $R$-module because $H \subseteq E$ (see [1], Exercise 18.10).

Let $\alpha: J \rightarrow R / H$ be an $R$-homomorphism, where again $J$ is the Jacobson radical of $R$. If $p=(0,0,0,1)$ then $p$ is an idempotent element of $R$ and $J=J p$. It follows that if $K$ is the right ideal containing $H$ such that $\operatorname{Im} \alpha=K / H$ then $K$ is contained in the ideal $(0, M, 0, T)$. For each element $x$ of $M$ choose an element $x^{M}$ of $M$ and an element $x^{T}$ of $T$ such that $\alpha(0, x, 0,0)=\left(0, x^{M}, 0, x^{T}\right)+H$. Since $\alpha$ is a homomorphism we note the following three facts.

(i) There exist elements $y_{0}$ in $Y$ and $t_{0}$ in $T$ such that $0^{M}=$ $y_{0}+m t_{0}, 0^{T}=t_{0}$. 
(ii) For all elements $x_{1}, x_{2}$ in $M$ there exist elements $y_{1}$ in $Y$ and $t_{1}$ in $T$ such that $\left(x_{1}+x_{2}\right)^{M}-x_{1}^{M}-x_{2}^{M}=y_{1}+m t_{1},\left(x_{1}+x_{2}\right)^{T}-$ $x_{1}^{T}-x_{2}^{T}=t_{1}$.

(iii) For all elements $x$ in $M$ and $c$ in $T$ there exist elements $y_{2}$ in $Y$ and $t_{2}$ in $T$ such that $(x c)^{M}-x^{M} c=y_{2}+m t_{2},(x c)^{T}-x^{T} c=t_{2}$. Define $\beta: M \rightarrow M / Y$ by $\beta(x)=\left(x^{M}-m x^{T}\right)+Y$ for every element $x$ of $M$. By (i), (ii) and (iii) $\beta$ is a $T$-homomorphism. But $Y=f M+X$ implies that $\operatorname{Ann}_{S}(Y)=\mathrm{Ann}_{S}(M)$. Therefore by hypothesis there exists an element $s_{1}$ of $S$ such that $\beta(x)=s_{1} x+Y(x \in M)$. Let $s$ be the element $\left(s_{1}, 0,0,0\right)$ of $R$. Then for each element $j$ of $J$ there exists an element $x$ of $M$ such that $j=(0, x, 0,0)$ and hence $\alpha(j)=$ $\left(0, x^{M}, 0, x^{T}\right)+H=s j+H$. Thus $\alpha(j)=s j+H(j \in J)$. By Corollary 2.6 and Lemmas 3.2-3.4 $R$ is a right CDPI-ring. This proves the theorem.

Combining Lemmas $3.1,3.2$ and Theorem 3.7 we have:

CoROllary 3.8. A ring $R$ is a semiprimary right CDPI-ring if and only if $R$ is semiprime Artinian or there exist semiprime Artinian rings $S$ and $T$ and a left $S$-, right $T$-bimodule $M$ such that $M$ is a faithful left $S$-module and for every $T$-submodule $X$ of $M$ such that $\operatorname{Ann}_{S}(X)=0$ and T-homomorphism $\varphi: M \rightarrow M / X$ there exists an element $a$ of $S$ with $\varphi(m)=a m+X$ for every $m$ in $M$, and $R$ is isomorphic to the ring $(S, M, 0, T)$.

COROLlaRy 3.9. In the above notation let $R$ be the semiprimary right SI-ring (S, $M, 0, T)$. Suppose that $R$ is a right CDPI-ring. Then there does not exist a left $S$-, right $T$-sub-bimodule $X$ of $M$ and a nonzero T-submodule $Y$ of $M$ such that $\operatorname{Ann}_{S}(X)=\operatorname{Ann}_{S}(M)$, $X \cap Y=0$ and $Y$ can be embedded in $X$.

Proof. Suppose that $M$ contains a sub-bimodule $X$ and a submodule $Y$ with the given properties. Let $X_{1}$ be a $T$-submodule of $X$ such that there is a $T$-isomorphism $\varphi: X_{1} \rightarrow Y$. Since $T$ is semiprime Artinian there exists a $T$-submodule $N$ of $M$ such that $M=X_{1} \oplus$ $Y \oplus N$. Define $\alpha: M \rightarrow M / X$ by $\alpha\left(x_{1}+y+n\right)=\varphi\left(x_{1}\right)+X$ for all $x_{1}$ in $X_{1}, y$ in $Y$ and $n$ in $N$. If $R$ is a right CDPI-ring then by the theorem there exists an element $s$ of $S$ such that for each element $x_{1}$ of $X_{1}, \varphi\left(x_{1}\right)+X=\alpha\left(x_{1}\right)=s x_{1}+X$. It follows that $\varphi\left(x_{1}\right) \in X \cap$ $Y=0$ for each element $x_{1}$ of $X_{1}$, a contradiction. Thus $R$ is not a right CDPI-ring.

CoROLlary 3.10. Suppose that $S$ and $T$ are simple rings and the above ring $R=(S, M, 0, T)$ is a right CDPI-ring. Then $M$ is a simple left $S$-, right $T$-bimodule. 
Proof. Let $X$ be a nonzero left $S$-, right $T$-sub-bimodule of $M$. Since $S$ is simple it follows that $\operatorname{Ann}_{S}(X)=\operatorname{Ann}_{S}(M)=0$. If $Y$ is a simple $T$-submodule of $M$ then $Y$ can be embedded in $X$, because $T$ is simple and simple right $T$-modules are isomorphic. By Corollary $3.9 X \cap Y \neq 0$ and hence $Y \subseteq X$. It follows that $X=M$.

We can express Corollary 3.10 in the following form.

COROLLARY 3.11. Let $R$ be a semiprimary right CDPI-ring with Jacobson radical $J$. If $R$ contains precisely two maximal ideals then $J$ is a minimal ideal of $R$.

4. Category equivalence. Let $R$ be a ring and $A, B$ be right $R$-modules. A monomorphism $\varphi: A \rightarrow B$ is called essential if and only if $\operatorname{Im} \varphi$ is an essential submodule of $B$; that is, $\operatorname{Im} \varphi \cap C \neq 0$ for every nonzero submodule $C$ of $B$. The first lemma in this section is elementary and well known but we shall include its proof for completeness.

Lemma 4.1. A right $R$-module $C$ is singular if and only if there exists an exact sequence $0 \rightarrow A \stackrel{\alpha}{\rightarrow} B \stackrel{\beta}{\rightarrow} C \rightarrow 0$ of right $R$-modules such that $\alpha: A \rightarrow B$ is an essential monomorphism.

Proof. Suppose that $C$ is singular. For each element $c$ of $C$ let $R_{c}=R$ and let $F=\bigoplus_{c} R_{c}$. Let $\pi: F \rightarrow C$ be the canonical projection. For each element $c$ of $C$ there exists an essential right ideal $E_{c}$ of $R=R_{c}$ such that $c E_{c}=0$. Let $E=\bigoplus_{c} E_{c}$. Then $E$ is an essential submodule of $F$ and $E \subseteq \operatorname{Ker} \pi$. If $K=\operatorname{Ker} \pi$ and $i: K \rightarrow F$ is inclusion then $0 \rightarrow K \stackrel{i}{\rightarrow} F \stackrel{\pi}{\rightarrow} C \rightarrow 0$ is an exact sequence such that $i$ is an essential monomorphism. Conversely, suppose that there exists an exact sequence $0 \rightarrow A \stackrel{\alpha}{\rightarrow} B \stackrel{\beta}{\rightarrow} C \rightarrow 0$ of right $R$-modules such that $\alpha$ is an essential monomorphism. Let $c \in C$ and let $b$ be an element of $B$ such that $\beta(b)=c$. It can easily be checked that $\operatorname{Ker} \beta=$ $\operatorname{Im} \alpha$ is an essential submodule of $B$ implies that $G=\{r \in R: b r \in$ $\operatorname{Ker} \beta\}$ is an essential right ideal of $R$. Also, $c G=\beta(b) G=\beta(b G)=0$. It follows that $C$ is singular.

CoRollary 4.2. A right $R$-module $C$ is a finitely generated singular module if and only if there exists an exact sequence $0 \rightarrow A \stackrel{\alpha}{\rightarrow} B \stackrel{\beta}{\rightarrow} C \rightarrow 0$ of right $R$-modules such that $B$ is finitely generated and $\alpha: A \rightarrow B$ is an essential monomorphism.

Lemma 4.3. A ring $R$ is a right RIC-ring if and only if every 
finitely generated singular right $R$-module is injective.

Proof. The sufficiency follows from the fact that if $E$ is an essential right ideal of $R$ then $R / E$ is a cyclic singular right $R$ module. Conversely, suppose that $R$ is a right RIC-ring. Let $n$ be a positive integer and $X$ a right $R$-module generated by elements $x_{1}, x_{2}, \cdots, x_{n}$. If $n=1$ there is nothing to prove. Suppose that $n>1$ and let $Y=x_{1} R+x_{2} R+\cdots+x_{n-1} R$. Then $Y$ is a singular module. If $Y$ is injective then there exists a submodule $Z$ of $X$ such that $X=Y \oplus Z$. It follows that $Z$ is a cyclic singular module and hence $Z$ is injective. Thus $X$ is injective. The result follows by induction on $n$.

CoRollary 4.4. Any ring Morita equivalent to a right RICring is itself a right RIC-ring.

Proof. By Corollary 4.2 since category equivalence preserves exact sequences, finitely generated modules and essential monomorphisms (see [1], Propositions 21.4, 21.6(5) and 21.8(2)).

THEOREM 4.5. A ring $R$ is a rihgt CEPI-ring if and only if every finitely generated right $R$-module is the extension of a projective right $R$-module by an injective right $R$-module.

Proof. The given condition is clearly sufficient for $R$ to be a right CEPI-ring. Conversely, suppose that $R$ is a right CEPI-ring. Let $n$ be a positive integer and $X$ be a right $R$-module generated by elements $x_{1}, x_{2}, \cdots, x_{n}$. If $n=1$ there is nothing to prove and so we suppose that $n>1$. Let $Y=x_{1} R+x_{2} R+\cdots+x_{n-1} R$. Suppose there is a submodule $A$ of $Y$ such that $A$ is projective and $Y / A$ is injective. Since $X / Y$ is cyclic and $R$ is a right CEPI-ring it follows that there exists a submodule $B$ of $X$ such that $Y \subseteq B, B / Y$ is projective and $X / B$ is injective. Now consider $B / A$. Since $Y / A$ is injective there exists a submodule $C$ of $B$ such that $A \subseteq C$ and $B / A=(Y / A) \oplus(C / A)$. Since $C / A \cong B / Y$ is projective and $A$ is projective it follows that $C \cong A \oplus(C / A)$ is projective. Moreover, $B / C \cong Y / A$ is injective and hence $X / C \cong(B / C) \oplus(X / B)$ is injective. The result follows by induction on $n$.

CoRollary 4.6. Any ring Morita equivalent to a right CEPIring is itself a right CEPI-ring.

Proof. By the theorem since category equivalence preserves exact sequences, finitely generated modules, projective modules and injective modules (see [1], Propositions 21.4, 21.6(2) and 21.8(2)). 
It is interesting to compare Theorem 2.5 with the next result.

THEOREM 4.7. A ring $R$ is a right SI-ring if and only if every right $R$-module is the extension of a projective right $R$-module by an injective right $R$-module.

Proof. Suppose that every right $R$-module is the extension of a projective module by an injective module. In particular, this means that $R$ is a right CEPI-ring. By [10], Lemma 2.4, $R$ is right nonsingular. Let $X$ be a singular right $R$-module. There exists a submodule $Y$ of $X$ such that $Y$ is projective and $X / Y$ is injective. Suppose that $Y \neq 0$ and let $y$ be a nonzero element of $Y$. Since $Y$ is projective there exists a homomorphism $\varphi: Y \rightarrow R$ such that $\varphi(y) \neq 0$. But there exists an essential right ideal $E$ of $R$ such that $y E=0$ and hence $\varphi(y) E=0$. This contradicts the fact that $R$ is right nonsingular. Thus $Y=0$ and $X$ is injective. It follows that $R$ is a right SI-ring.

Conversely, suppose that $R$ is a right SI-ring. Let $A$ be a right $R$-module and $\mathfrak{A}$ the collection of cyclic submodules of $A$. By Zorn's lemma there is a maximal collection $\mathfrak{B}$ of members of $\mathfrak{A}$ whose sum is direct. Let $A$ be an index set and $x_{\lambda}$ elements of $A$ such that $\mathfrak{B}$ is the collection of submodules $x_{\lambda} R(\lambda \in \Lambda)$. Let $B=\bigoplus_{A} x_{\lambda} R$. The choice of $B$ ensures that $B$ is an essential submodule of $A$. Since $R$ is a right SI-ring it follows that $R$ is right hereditary (see [5], Proposition 3.3) and hence $B$ is projective. Moreover $A / B$ is a singular right $R$-module and is injective because $R$ is a right SI-ring. It follows that every right $R$-module is the extension of a projective module by an injective module.

COROLLARY 4.8. If $R$ is a right Noetherian right RIC-ring then every right $R$-module is the extension of a projective right $R$ module by an injective right $R$-module.

Proof. By the theorem and [10], Theorem 4.1.

In particular Corollary 4.8 tells us that any right Noetherian right CDPI-ring $R$ has the property that every right $R$-module is the extension of a projective module by an injective module.

Next we consider right FGDPI-rings. The proof of Corollary 4.6 gives immediately:

Lemma 4.9. Any ring Morita equivalent to a right FGDPI-ring is itself a right FGDPI-ring. 
Before examining the relationship between right FGDPI-rings and right CDPI-rings we first introduce some notation. Let $R$ be a ring, $n$ a positive integer and $R_{n}$ the complete ring of $n \times n$ matrices with entries in $R$. Let $\left(r_{i j}\right)$ denote the $n \times n$ matrix whose $(i, j)$ th entry is the element $r_{i j}$ or $R$. For any right $R$-module $X$ let $X^{(n)}$ denote the right $R$-module $X \oplus X \oplus \cdots \oplus X$ ( $n$ copies). Then $X^{(n)}$ can be made into an $R_{n}$-module by defining:

$$
\left(x_{1}, x_{2}, \cdots, x_{n}\right)\left(r_{i j}\right)=\left(\sum_{k=1}^{n} x_{k} r_{k 1}, \sum_{k=1}^{n} x_{k} r_{k 2}, \cdots, \sum_{k=1}^{n} x_{k} r_{k n}\right),
$$

where $x_{i} \in X$ and $r_{i j} \in R(1 \leqq i, j \leqq n)$. Let $e_{i j}$ denote the matrix unit in $R_{n}$ with 1 in the $(i, j)$ th position and zeros elsewhere. For any right $R_{n}$-module $Y, Y e_{11}$ is a right $R$-module. It is easy to check that for any right $R$-module $X$ the right $R$-modules $X$ and $X^{(n)} e_{11}$ are isomorphic. Recall the following result.

LeMma 4.10 (See [7], Corollary 2.3). With the above notation, a right $R_{n}$-module $X$ is projective (respectively injective) if and only if the right $R$-module $X e_{11}$ is projective (respectively injective).

THEOREM 4.11. Let $n$ be a positive integer. A ring $R$ is a right $F G D P I_{n}$-ring if and only if $R_{n}$ is a right CDPI-ring.

Proof. Suppose that $R_{n}$ is a right CDPI-ring. Let $X$ be a right $R$-module generated by elements $x_{1}, x_{2}, \cdots, x_{n}$. If $Y=X^{(n)}$ then $Y$ is the cyclic right $R_{n}$-module $\left(x_{1}, x_{2}, \cdots, x_{n}\right) R_{n}$. There exists a projective right $R_{n}$-module $P$ and an injective right $R_{n}$-module $Q$ such that $Y=P \oplus Q$. Then $Y e_{11}=\left(P e_{11}\right) \oplus\left(Q e_{11}\right)$, as $R$-modules. Since the right $R$-modules $X$ and $Y e_{11}$ are isomorphic it follows that $X$ is the direct sum of a projective module and an injective module by Lemma 4.10. Thus $R$ is a right $\mathrm{FGDPI}_{n}$-ring.

Conversely, suppose that $R$ is a right FGDPI $_{n}$-ring. Let $A=a R_{n}$ be a cyclic right $R_{n}$-module. Then $A e_{11}=a R_{n} e_{11}=\sum_{k=1}^{n} a e_{k 1} R$ is an $n$-generator right $R$-module. By hypothesis there exists a projective right $R$-module $B$ and an injective right $R$-module $C$ such that $A e_{11}=B \oplus C$. Now $R_{n}=R_{n} e_{11} R_{n}$ implies that $A e_{11} R_{n}=A R_{n} e_{11} R_{n}=A$ and hence $A=\left(B R_{n}\right)+\left(C R_{n}\right)$. Since $B=B e_{11}$ and $C=C e_{11}$ it follows that

$$
B R_{n}=\sum_{k=1}^{n} B e_{1 k} \quad \text { and } \quad C R_{n}=\sum_{k=1}^{n} C e_{1 k} .
$$

It can easily be checked that $B \cap C=0$ implies that $\left(B R_{n}\right) \cap\left(C R_{n}\right)=0$. That is $A=\left(B R_{n}\right) \oplus\left(C R_{n}\right)$. Moreover, $\left(B R_{n}\right) e_{11}=B$ and $\left(C R_{n}\right) e_{11}=C$. By Lemma $4.10 B R_{n}$ is a projective right $R_{n}$-module and $C R_{n}$ is 
an injective right $R_{n}$-mudule. It follows that $R_{n}$ is a right CDPIring.

CoROLlaRy 4.12. A ring $R$ is a right FGDPI-ring if and only if $R_{n}$ is a right CDPI-ring for every positive integer $n$.

It is interesting to contrast Theorem 4.11 with the next result.

THEOREM 4.13. Let $R$ be a right CDPI-ring and $e$ be an idempotent element of $R$ such that $R=R e R$. Then the subring eRe of $R$ is a right CDPI-ring.

Proof. Let $S$ denote the ring $e R e$ and let $I$ be a right ideal of $S$. If $J$ is the right ideal $I R$ of $R$ then $J \subseteq e R$ since $I=e I$. By hypothesis there exist right ideals $F$ and $G$ of $R$ such that $J \subseteq$ $F \leqq e R, J \subseteq G \leqq e R, F / J$ is a projective right $R$-module, $G / J$ is an injective right $R$-module and $e R / J=(F / J) \oplus(G / J)$. Since $e R / G \cong F / J$ is projective there exists a right ideal $H$ of $R$ such that $e R=G \oplus H$. Then $G e$ and $H e$ are right ideals of $S, S=(G e) \oplus(H e)$ and hence $S /(G e)$ is a projective right $S$-module. Moreover, $e R=F+G$, $F \cap G=J$ together imply $S=(F e)+(G e)$ and $(F e) \cap(G e)=J e=I R e=$ $I e R e=I$. Thus $S / I$ is the direct sum $((F e) / I) \oplus((G e) / I)$ of the right $S$-modules $(F e) / I$ and $(G e) / I$. Also, $(F e) / I \cong S /(G e)$ is a projective right $S$-module. It remains to prove that $(G e) / I$ is an injective right $S$-module. Note that $G=G R=G R e R=G e R$. Thus it is sufficient to prove the following result.

LeMMA 4.14. Let $R$ be a ring and $e$ be an idempotent element of $R$ such that $R=$ ReR. Let $A \subseteq B$ be right ideals of the ring $S=e R e$ and $\bar{A}=A R, \bar{B}=B R$. If $\bar{B} / \bar{A}$ is an injective right $R$ module then $B / A$ is an injective right $S$-module.

Proof. Let $C$ be a right ideal of $S$ and $\varphi: C \rightarrow B / A$ an $S$-homomorphism. Let $V$ be a set of coset representatives of $A$ in $B$ and define a mapping $\alpha: C \rightarrow V$ by $\alpha(c)+A=\varphi(c) \quad(c \in C)$. Define $\bar{\varphi}$ : $C R \rightarrow \bar{B} / \bar{A}$ by

$$
\bar{\varphi}\left(\sum_{i=1}^{n} c_{i} r_{i}\right)=\sum_{i=1}^{n} \alpha\left(c_{i}\right) e r_{i}+\bar{A}
$$

for all positive integers $n$ and elements $c_{i}$ of $C$ and $r_{i}$ of $R(1 \leqq i \leqq n)$. Clearly $\bar{\phi}$ is independent of the choice of $V$. Suppose $n$ is a positive integer, $r_{i} \in R$ and $c_{i} \in C(1 \leqq i \leqq n)$ and

$$
\sum_{i=1}^{n} c_{i} r_{i}=0 \text {. }
$$


For any element $x$ of $R$,

$$
\sum_{i=1}^{n} c_{i} e r_{i} x e=0
$$

and hence

$$
\sum_{i=1}^{n} \varphi\left(c_{i}\right) e r_{i} x e=0
$$

That is, for all $x$ in $R$,

$$
\sum_{i=1}^{n} \alpha\left(c_{i}\right) e r_{i} x e \in A .
$$

Since $R=R e R$ it follows that $1 \in R e R$ and hence

$$
\sum_{i=1}^{n} \alpha\left(c_{\imath}\right) e r_{i} \in A R=\bar{A} \text {. }
$$

Thus $\bar{\varphi}$ is well defined and clearly $\bar{\phi}$ is an $R$-homomorphism. By hypothesis there exists an element $b$ of $\bar{B}$ such that $\bar{\varphi}(r)=b r+\bar{A}$ $(r \in C)$. It follows that $b e \in \bar{B} e=B R e=B e R e=B$. Let $c \in C$. Then $c=c e=e c$ and $\varphi(c)=\alpha(c)+A=\alpha(c) e+A$ and $\bar{\varphi}(c)=\alpha(c) e+\bar{A}=$ $b c+\bar{A}=b e c+\bar{A}$. This implies that $\alpha(c) e-b e c \in \bar{A} \cap S=A$ and hence $\varphi(c)=b e c+A$. Thus $\varphi(c)=b e c+A(c \in C)$. It follows that $B / A$ is an injective right $S$-module. This completes the proof of Lemma 4.14 and hence also of Theorem 4.13.

5. Right FGDPI-rings, Let $R$ be a semiprime right Goldie ring. Goldie [4], Theorems 4.1 and 4.4, proved that $R$ has a (classical) right quotient ring $Q$ and $Q$ is semiprime Artinian. Levy [7], Theorem 5.3, proved that if $R$ has the additional property that every finitely generated torsion-free right $R$-module is a submodule of a free right $R$-module then $Q$ is the left quotient ring of $R$ and hence by [4], Theorem 4.4, $R$ is a left Goldie ring. In actual fact to prove that $Q$ was the left quotient ring of $R$ all Levy needed was the fact that every 2-generator right $R$-submodule of $Q$ is contained in a free right $R$-module. Thus we can state Levy's result in the following form.

LEMMA 5.1. Let $R$ be a semiprime ring Goldie ring with right quotient ring $Q$ such that every 2-generator right $R$-submodule of $Q$ is contained in a free right $R$-module. Then $R$ is a left Goldie ring.

Next we restate [7], Theorem 6.1, as follows.

LEMMA 5.2. Let $R$ be a semiprime right and left Goldie right 
(and left) semihereditary ring. Then every finitely generated right $R$-module $X$ is the direct sum of its singular submodule $Z(X)$ and a projective $R$-submodule $P$.

COROLLARY 5.3. Let $R$ be a semiprime right and left Goldie ring. Then $R$ is a right FGDPI-ring if and only if $R$ is a right RIC-ring.

Proof. The necessity follows by [10], Lemma 2.4. Conversely, suppose that $R$ is a right RIC-ring. Let $X$ be a finitely generated right $R$-module with singular submodule $Z$. By [10] Corollary 4.3 and Lemma 4.4, $R$ is right semihereditary. By Lemma 5.2 there exists a projective submodule $P$ of $X$ such that $X=Z \oplus P$. By Lemma $4.3 Z$ is injective. It follows that $R$ is a right FGDPI-ring.

Let $R$ be a semiprime right Noetherian ring with right quotient ring $Q$ and suppose $Q$ is a finitely generated right $R$-module. Let $a$ be a regular element of $R$ and consider the ascending chain $a^{-1} R \cong a^{-2} R \cong a^{-3} R \cong \cdots$ of $R$-submodules of $Q$. Since $Q$ is a Noetherian right $R$-module there exists a positive integer $n$ such that $a^{-n} R=a^{-n-1} R$. Then $a^{-n-1}=a^{-n} b$ for some element $b$ of $R$ and hence $1=a b=b a$. It follows that $R=Q$.

LEMMA 5.4. Let $R$ be a prime right Noetherian right FGDPI $I_{2}$ ring. Then $R$ is a left Goldie ring.

Proof. Let $Q$ be the right quotient ring of $R$. In view of Lemma 5.1 it is sufficient to prove that every 2-generator right $R$ submodule of $Q$ is contained in a free right $R$-module. Let $X$ be a 2 -generator right $R$-submodule of $Q$. By hypothesis there exists a projective $R$-submodule $P$ of $X$ and an injective $R$-submodule $I$ of $X$ such that $X=P \oplus I$. Suppose that $I \neq 0$. For any regular element $c$ of $R$ we have $I=I c$ (see [7], Theorem 3.1). Since $I$ is torsion-free, for all elements $x$ of $I$ and regular elements $c$ of $R$ there exists a unique element $x$ of $I$ such that $\bar{x} c=x$. By defining $x c^{-1}=\bar{x}$ for all $x$ in $I$ and $c$ regular in $R$ we can make $I$ into a right $Q$-module. Since $I \neq 0$ and $Q$ is simple Artinian it follows that $I$ contains a simple right $Q$-module. Since $Q$ is simple Artinian all simple right $Q$-modules are isomorphic. Because $I$ is a finitely generated right $R$-module it follows that $Q$ is a finitely generated right $R$-module. As our remarks above show, in this case $R=Q$ and hence $R$ is left Goldie. Now suppose that $Q \neq R$. Then $I=0$, $X=P$ and hence $X$ is contained in a free right $R$-module. Thus every 2-generator right $R$-submodule of $Q$ is contained in a free right $R$-module. By Lemma $5.1 R$ is a left Goldie ring. 
LeMMA 5.5. Let $S$ and $T$ be subrings of a ring $R$ such that $R=S \oplus T$. Let $n$ be a positive integer. Then $R$ is a right $F G D P I_{n}-$ ring if and only if $S$ and $T$ are both right $F G D P I_{n}$-rings.

Proof. Suppose that $R$ is a right FGDPI $_{n}$-ring. Let $X$ be an $n$-generator right $S$-module. We can make $X$ into an $n$-generator right $R$-module by defining $x(s+t)=x s$ for all $x$ in $X, s$ in $S$ and $t$ in $T$. By hypothesis there exists a projective right $R$-module $P$ and an injective right $R$-module $I$ such that $X=P \oplus I$. It can easily be checked that $P$ is a projective right $S$-module and $I$ is an injective right $S$-module. It follows that $S$ is a right FGDPI $_{n}$-ring. Similarly $T$ is a right $\mathrm{FGDPI}_{n}$-ring.

Conversely, suppose first that $n=1$; that is, $S$ and $T$ are both right CDPI-rings. Let $E$ be a right ideal of $R=S \oplus T$. Then there exists a right ideal $E_{1}$ of $S$ and a right ideal $E_{2}$ of $T$ such that $E=E_{1} \oplus E_{2}$. Since $S$ and $T$ are right CDPI-rings there exist idempotent elements $e_{1}$ of $S$ and $e_{2}$ of $T$ such that $E_{1} \subseteq e_{1} S, E_{2} \subseteq e_{2} T$, $A=\left(e_{1} S\right) / E_{1}$ is an injective right $S$-module and $B=\left(e_{2} T\right) / E_{2}$ is an injective right $T$-module. The Abelian group $C=A \oplus B$ can be made into a right $R$-module by defining $(a, b)(s+t)=(a s, b t)$ for all $a$ in $A, b$ in $B, s$ in $S$ and $t$ in $T$. If $f=e_{1}+e_{2}$ then $f$ is an idempotent element of $R$ and $E \cong f R$. Moreover, $(f R) / E$ is isomorphic to the right $R$-module $C$. If $F$ is a right ideal of $R$ then $F=F_{1} \oplus F_{2}$ for some right ideals $F_{1}$ of $S$ and $F_{2}$ of $T$, and it can easily be checked that any $R$-homomorphism $\varphi: F \rightarrow C$ can be lifted to an $R$-homomorphism $\bar{\varphi}: R \rightarrow C$. Thus $C$ is injective. It follows that $R$ is a right CDPI-ring. Now suppose that $n$ is any positive integer and $S$ and $T$ are both right FGDPI $_{n}$-rings. By Theorem 4.11 the matrix rings $S_{n}$ and $T_{n}$ are right CDPI-rings. But clearly $R_{n} \cong S_{n} \oplus T_{n}$ and the above argument shows that $R_{n}$ is a right CDPI-ring. By Theorem $4.11 R$ is a right FGDPI $_{n}$-ring.

It is clear that one consequence of Lemma 5.5 is the following result.

CoRollary 5.6. Let $S$ and $T$ be subrings of a ring $R$ such that $R=S \oplus T$. Then $R$ is a right FGDPI-ring if and only if both $S$ and $T$ are right FGDPI-rings.

THEOREM 5.7. Let $R$ be a semiprime right Noetherian ring. Then the following statements are equivalent.

(i) $R$ is a right $F G D P I_{2}$-ring.

(ii) $R$ is a right FGDPI-ring.

(iii) $R$ is a left Goldie right RIC-ring. 
(iv) $\quad R$ is a finite direct sum $A \oplus B_{1} \oplus B_{2} \oplus \cdots \oplus B_{n}$ where $A$ is a semiprime Artinian ring and for each $1 \leqq i \leqq n$ the ring $B_{i}$ is a simple right and left Noetherian ring Morita equivalent to a Noetherian simple PCI-domain.

Proof. (ii) $\Rightarrow$ (i) is clear. (iii) $\Rightarrow$ (ii) is a consequence of Corollary 5.3. (iv) $\Rightarrow$ (iii) is a consequence of [5], Theorem 3.11. It remains to prove (i) $\Rightarrow$ (iv). Suppose that $R$ is a right FGDPI $_{2}$-ring. By [5], Theorem 3.11, $R$ is a finite direct sum $A \oplus B_{1} \oplus B_{2} \oplus \cdots \oplus B_{n}$ where $A$ is semiprime Artinian and $B_{i}$ is a simple right Noetherian ring Morita equivalent to a right Noetherian simple right PCI-domain $D_{i}$ for each $1 \leqq i \leqq n$. By Lemmas 5.4 and 5.5 the ring $B_{i}$ is a left Goldie ring for each $1 \leqq i \leqq n$. Thus, for each $1 \leqq i \leqq n, D_{i}$ is left Goldie and hence a Noetherian simple PCI-domain by [3], Theorem 22 and subsequent remarks. It follows that $B_{i}$ is left Noetherian $(1 \leqq i \leqq n)$. This proves (iv).

CoROLlaRY 5.8. For any positive integer $m$ a ring $R$ is a right Noetherian right $F G D P I_{m}$-ring if and only if $R$ is a finite direct sum $A \oplus B_{1} \oplus B_{2} \oplus \cdots \oplus B_{n}$ where $A$ is a right Artinian right $F G D P I_{m}$-ring and the ring $B_{i}$ is a simple right and left Noetherian ring Morita equivalent to a Noetherian simple PCI-domain for each $1 \leqq i \leqq n$.

Proof. By the theorem and Lemma 5.5.

CoRollary 5.9. Let $R$ be a semiprime ring. Then the following statements are equivalent.

(i) $R$ is a right Noetherian right $F G D P I_{2}$-ring.

(ii) $R$ is a left Noetherian left $F G D P I_{2}$-ring.

(iii) $R$ is a right Noetherian right FGDPI-ring.

(iv) $R$ is a left Noetherian left FGDPI-ring.

Proof. By the theorem, Lemma 5.5 and Corollary 5.6.

CoROLlaRY 5.10. Let $R$ be a right Noetherian right FGDPI $I_{2}^{-}$ ring with Jacobson radical $J$. Then the ring $R / J$ is a left Noetherian left FGDPI-ring. Moreover $R$ is a left SI-ring and in particular $R$ is left hereditary.

Proof. By Corollary $5.8 R / J$ is a right Noetherian right FGDPI $_{2}$ ring and by Corollary $5.9 R / J$ is a left Noetherian left $\mathrm{FGDPI}_{2}$-ring. In $\S 1$ we noted that right Noetherian right CDPI-rings are right SI-rings. Also by [5], Proposition 3.5, right Artinian right SI-rings 
are left SI-rings. The result follows by [5], Theorem 3.11 and Proposition 3.3.

\section{REFERENCES}

1. F. W. Anderson and K. R. Fuller, Rings and categories of modules, (Springer-Verlag, 1974).

2. J. H. Cozzens, Homological properties of the ring of differential polynomials, Bull. Amer. Math. Soc., 76 (1970), 75-79.

3. C. Faith, When are proper cyclics injective? Pacific J. Math., 45 (1973), 97-112.

4. A. W. Goldie, Semiprime rings with maximum condition, Proc. London Math. Soc.,

(3) 10 (1960), 201-220.

5. K. R. Goodearl, Singular torsion and the splitting properties, Amer. Math. Soc. Memoirs, 124 (1972).

6. I. Kaplansky, Rings of operators, (University of Chicago, 1955).

7. L. Levy, Torsion-free and divisible modules over non-integral domains, Canad. J. Math., 15 (1963), 132-151.

8. B. L. Osofsky, Non-injective cyclic modules, Proc. Amer. Math. Soc., 19 (1968), 1383-1384.

9. L. W. Small,Semihereditary rings, Bull. Amer. Math. Soc., 73 (1967), 656-658.

10. P. F. Smith, Rings characterized by their cyclic modules, to appear in Canad. J. Math.

Received May 4, 1977.

UNIVERSITY OF GLASGOW

UnIVERSITY GARDENS

GLASGOW G12 8QW

SCOTLAND 


\section{PACIFIC JOURNAL OF MATHEMATICS}

\section{EDITORS}

RICHARD ARENS (Managing Editor)

University of California

Los Angeles, California 90024

C. W. CURTIS

University of Oregon

Eugene, OR 97403

C. C. MOORE

University of California

Berkeley, CA 94720

\section{J. DUGUNDJI}

Department of Mathematics University of Southern California Los Angeles, California 90007

R. Finn aNd J. Milgram Stanford University Stanford, California 94305

\section{ASSOCIATE EDITORS}

E. F. BeCKenbaCH

B. H. NeumanN

F. WOLF

K. YosHIDA

\section{SUPPORTING INSTITUTIONS}

UNIVERSITY OF BRITISH COLUMBIA CALIFORNIA INSTITUTE OF TECHNOLOGY UNIVERSITY OF CALIFORNIA MONTANA STATE UNIVERSITY UNIVERSITY OF NEVADA, RENO NEW MEXICO STATE UNIVERSITY OREGON STATE UNIVERSITY UNIVERSITY OF OREGON
UNIVERSITY OF SOUTHERN CALIFORNIA STANFORD UNIVERSITY UNIVERSITY OF HAWAII UNIVERSITY OF TOKYO UNIVERSITY OF UTAH WASHINGTON STATE UNIVERSITY UNIVERSITY OF WASHINGTON 


\section{Pacific Journal of Mathematics}

\section{Vol. 76, No. $1 \quad$ November, 1978}

Ata Nuri Al-Hussaini, Potential operators and equimeasurability ......... 1

Tim Anderson and Erwin Kleinfeld, Semisimple nil algebras of type $\delta . \ldots .99$

Stephen LaVern Campbell, Linear operators for which $T^{*} T$ and $T+T^{*}$

commute. III ......................................

Robert Jay Daverman, Special approximations to embeddings of codimension one spheres...............................

Donald M. Davis, Connective coverings of $\mathrm{BO}$ and immersions of projective

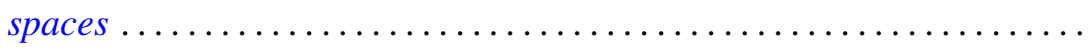

V. L. (Vagn Lundsgaard) Hansen, The homotopy type of the space of maps of

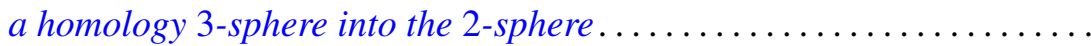

James Victor Herod, A product integral representation for the generalized

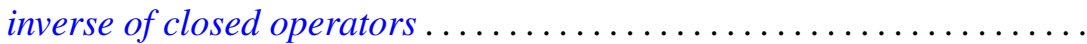

A. A. Iskander, Definability in the lattice of ring varieties ..............

Russell Allan Johnson, Existence of a strong lifting commuting with a

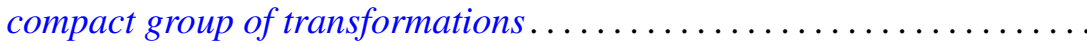

Heikki J. K. Junnila, Neighbornets...................... 83

Klaus Kalb, On the expansion in joint generalized eigenvectors . ......... 109

F. J. Martinelli, Construction of generalized normal numbers . . . . . . . . . 117

Edward O'Neill, On Massey products ....................... 123

Vern Ival Paulsen, Continuous canonical forms for matrices under unitary

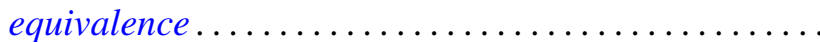

Justin Peters and Terje Sund, Automorphisms of locally compact groups . . . 143

Duane Randall, Tangent frame fields on spin manifolds . . . .

Jeffrey Brian Remmel, Realizing partial orderings by classes of co-simple sets . . . .

J. Hyam Rubinstein, One-sided Heegaard splittings of 3-manifolds ...

Donald Charles Rung, Meier type theorems for general boundary approach

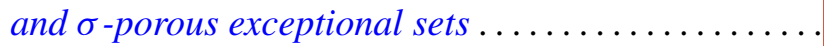

Ryōtarō Satō, Positive operators and the ergodic theorem

Ira H. Shavel, A class of algebraic surfaces of general type constructed from

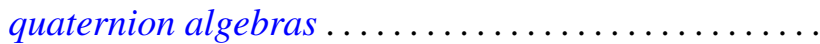

Patrick F. Smith, Decomposing modules into projectives and injectives ....

Sergio Eduardo Zarantonello, The sheaf of outer functions in the polydisc... 